\title{
Rapid Nucleic Acid Analysis for Contaminant Impact Evaluation
}

Oak Ridge National Laboratory

May 25,2001

Annual Report

\section{Principal Investigator}

Dr. Winston Chung-Hsuan Chen

(423)-574-6895 (Phone)

(423)-576-2115 (Fax)

CHENC@ORNL.GOV

Oak Ridge National Laboratory

P. O. Box 2008

Oak Ridge, TN. 37831-6378

\section{Other Contributors:}

Dr. Kai-Lin Lee

Dr. Narayana Isola

Dr. Chao-Yang Liu

Prof. Karla J Matteson

Prof. Lauri Sammartano 


\section{Research Objective:}

The objective of this program is to develop innovative DNA detection technologies to achieve fast mutation screening and to reveal the linkage between gene mutation and contaminants. The specific approach are (1) to develop innovative multiplexing hybridization detection for DNA mutation detection,

(2) to develop sequence-proof microarray hybridization technology (3) to develop hybridization on disk and

(4) to apply these new DNA detection technology for mutation analysis of contaminated fish and to validate the pollution-mediated mutation can be used for sound risk analysis for setting up the priorities for waste cleanup.

\section{Research Progress and Implication:}

During the past year, we have significant achievement in mass spectrometry DNA analysis for hybridization. Since this program is to address the DNA mutation due to the exposure to contaminated media and to promote a better understanding of the relationship between exposure and health impact, high throughput DNA analysis technology is critically needed. During the past few years, microarray hybridization has been considered as a candidate for high throughput DNA analysis. However, there are two disadvantages with present hybridization detection technology. At present, all hybridization-on-chip is detected by laser induced fluorescence. DNA probes need to be tagged with dye molecules. At each hybridization site, only single hybridization reaction can be pursued. However, with mass spectrometry for microarray hybridization detection, no tagging is needed. In addition, multiplexing hybridization can be achieved. Thus, the speed can be one order of magnitude faster and the cost is also significantly less.

The major achievements during the past year are listed and discussed in the follows.

\section{(1) Successful detection of microarray hybridization with laser desorption mass} spectrometry:

Most detections of hybridization at present are based on laser induced fluorescence or radioactivity. Labeling of DNA is often needed. In most work, polymerase chain reaction (PCR) is needed to amplify target DNA for hybridization. During the past year, we succeeded in hybridization detection with mass spectrometry not only with PCR products but also genomic DNAs. We have bound genomic DNA from M13 and lambda bacteriophage onto nylon membranes and hybridized these membranes with oligonucleotides complementary to the two bacteriophages. The presence of oligonuclotides was detected by direct in situ mass spectrometry and successful hybridizations were obtained. The three oligonucleotide probes used were readily detected and resolved. This promises to be a useful non-radioactive method. The two probes used for Lambda phage were 25 mer oligonucleotides and could be resolved as two separate peaks clearly indicating that the resolving power of mass spectrometry for analysis of modified oligonucleotide in short sequences.

(2) Laser desorption mass spectrometry for multiplexing hybridization detection.

Since there are between 50,000 to 100,000 genes in human genome and each gene can have more than hundreds of different mutations, unusual high throughput DNA analysis will be needed to evaluate mutation due to the environmental impact. At present, all the 
available hybridization detection can only have one hybridization reaction at one site.

Recently, we demonstrated the successful multiplexing hybridization detection with laser desorption mass spectrometry. Six different hybridization reaction with probes tagged with different molecular weights have been detected at a single hybridization site. We expect this technology can lead a quantum jump in term of the analysis speed in the future.

\section{Planned activities:}

The following tasks will be pursued during the coming year:

(1) To develop the technology to distinguish the single-base-mismatched hybridization from a perfectly complementary hybridization.

(2) To apply hybridization technology to gene mutation.

(3) To evaluate the mutation due to environmental impact

(4) To pursue high throughput DNA analysis with microarray arrangement with mass spectrometry detection

\section{Publications:}

(1) N. R. Isola, S. L. Allman, V. V. Golovlev and C. H. Chen " MALDI-TOF Mass Spectrometric Method for Detection of Complementary Nucleic Acids “ Analytical Chemistry (in Press)

(2) V. V. Golovlev, S. H. Lee, S. L. Allman, N. I. Taranenko and C. H. Chen, “ Nonresonance Matrix-assisted Laser Desorption/IOnization of Oligonucleotide: Mechnaism of Ion Desorption”, Anal. Chem. 73, 809-812 (2001)

(3) N. I. Taranenko, R. Hurt, J. Z. Zhou, N. R. Isola, H. Huang, S. H. Lee, and C. H. Chen, "Laser desorption mass spectrometry for microbial DNA analysis" Environmental Technology (submitted) 